\title{
Impact of laser on bismuth thin-films
}

\section{A 10 ps time-resolved study at the CRISTAL diffraction beamline (SOLEIL synchrotron)}

\author{
C. Laulhé1,2,a, M. Cammarata ${ }^{3}$, M. Servol ${ }^{3}$, R.J. Dwayne Miller ${ }^{4}$, M. Hada ${ }^{4}$, and S. \\ Ravy $^{2}$ \\ 1 Université Paris-Sud, 91405 Orsay Cedex, France \\ 2 Synchrotron SOLEIL, L'Orme des merisiers Saint Aubin - BP48, 91192 Gif-sur-Yvette \\ Cedex, France \\ 3 Institut de Physique de Rennes, UMR 6251 UR1-CNRS, Bat 11A Campus de Beaulieu, \\ University Rennes 1, Rennes, France \\ 4 Max Planck Research Department for Structural Dynamics at the Center for Free Electron \\ Laser Science, University of Hamburg c/o DESY, Luruper Chaussee 149, 22761 Hamburg, \\ Germany
}

\begin{abstract}
We used the newly developed low- $\alpha$ mode of SOLEIL synchrotron to observe optically induced strain waves in $200 \mathrm{~nm}$ bismuth thin-films.
\end{abstract}

\section{Introduction}

Structural behaviors of crystals after a femtosecond near-infrared laser pulse cover a large number of phenomena, with time constants spanning at least ten orders of magnitude, from a few hundreds of fs $\left(\sim 10^{-13} \mathrm{~s}\right)$ to the ms $\left(10^{-3} \mathrm{~s}\right)$ [1]. This remarkable range is due to the different physical phenomena at play in the whole dynamical process before complete relaxation: electron excitation, electron thermalization, electron-phonon interactions, optical and acoustic mode propagations or strain pulse propagations, and heat diffusion.

All these phenomena can now be tracked through developments of the pumpprobe scheme. With this technique, the effect of the laser pulse (the pump) is probed in a stroboscopic way after a certain delay by another light pulse (the probe), the photon energy of which determining the information that can be obtained. The pump pulse usually belongs to the infrared-visible region of the electromagnetic spectrum. Using another infrared or visible pulse as the probe allows one to measure the transient reflectivity (or transmission) and to obtain information about valence electrons. Ultra violet pulses can be used to eject photoelectrons and probe the band structure as a function of time. To get information on the structure dynamics, diffraction of electron or hard X-ray pulses has to be used. The advent of new X-ray sources have impulsed the development of time-resolved diffraction methods, which are now routinely performed.

\footnotetext{
a e-mail: laulhe@synchrotron-soleil.fr
} 
It is established that third generation synchrotron sources are ideally placed for studies with sub-ns temporal resolution. Indeed, electron bunches that circulate in storage rings naturally give rise to X-ray pulses with slightly less than 100 ps full width at half maximum (FWHM). Recently, X-ray pulse durations in the ps range have also been obtained at synchrotrons, by using either low- $\alpha$ modes of operation [2-4] or crab cavities [5]. For ultrashort X-ray pulses with 100 fs duration or less, several types of sources are currently available: laser-slicing at synchrotrons $[6,7]$, laser-driven plasma sources [8], laser-driven plasma accelerators [9], and free electron lasers [11]. The latter facilities are currently providing the most intense and shortest ( $\sim 10 \mathrm{fs}$ ) hard X-ray pulses ever seen [11]. Still, synchrotrons are able to provide fairly intense pulses with few ps durations, giving a time resolution well adapted to the study of coherent excitations of acoustic modes [12] and strain pulse propagation in samples with dimensions in the nm range [13-15].

In this article, we demonstrate the use of $10 \mathrm{ps} \mathrm{X}$-ray pulses produced in the newly-developed low- $\alpha$ mode at SOLEIL, for time-resolved diffraction experiments at the CRISTAL beamline. We apply the technique to observe the propagation of a laser-induced strain pulse in a bismuth thin-film of thickness $L=200 \mathrm{~nm}$. With its simple rhombohedral structure [16] and its 83 electrons per atom, which makes it a strong X-ray scatterer, bismuth is ideally suited for diffraction experiments. It is also known as the first material in which laser-induced coherent phonons were observed by using pump-probe reflectivity [17] and, later, X-ray diffraction [18-20].

Upon laser excitation of bismuth, some of the valence electrons are promoted to the higher energy bands, which changes the intra-atomic potential and subsequently triggers coherent optical phonon modes [21]. The "hot" electrons then relax in a few ps [20]. This transfer of energy to the lattice leads to a temperature increase in the laser penetration depth of the bismuth thin film (26 nm [22]). A longitudinal stress is thus generated which is relaxed through a strain pulse propagating with the speed of sound [23] and boucing at the substrate-film and air-film interfaces. In the following, we report on the measurement of the resulting oscillations of average longitudinal strain, with a time resolution of about $10 \mathrm{ps}$.

\section{Experimental}

\subsection{Newly developed "low- $\alpha$ " mode for ps time-resolved studies at SOLEIL}

The standard mode of operation for SOLEIL's storage ring is based on a hybrid filling pattern of electrons, consisting in a $5 \mathrm{~mA}$ single bunch diametrically opposed to a $425 \mathrm{~mA}$ multi-bunch beam spread over $3 / 4$ of the storage ring circumference. The temporal spacing between the isolated bunch and each of its two first neighbors equals $147 \mathrm{~ns}$, which allows for time-resolved pump-probe experiments using choppers or gated detectors. The X-ray pulses that are emitted by the $5 \mathrm{~mA}$ isolated bunch have a duration of 59 ps FWHM and easily provide a sub-ns temporal resolution.

The SOLEIL synchrotron also runs in modes especially optimized for time-resolved studies. In the 8 -bunch mode, 8 equidistant $12.5 \mathrm{~mA}$ bunches are circulating in the storage ring with a time interval of $147 \mathrm{~ns}$. In the related single-bunch mode, the ring is filled with a single bunch of $10 \mathrm{~mA}$. Thanks to the high currents carried by the isolated bunches, these modes provide intense X-ray pulses with a duration kept limited to about 70 ps FWHM. At the CRISTAL diffraction beamline, the number of photons per X-ray pulse at $7.15 \mathrm{keV}$ in a $10^{-4}$ bandwidth is estimated to $610^{5}$.

Recently, a so-called "low- $\alpha$ " mode of operation has been implemented at SOLEIL, in order to obtain X-ray pulses with few ps duration $[3,4]$. The key point is to decrease the electron bunch lengths, to which the X-ray pulse durations are directly 
proportional. In the "low- $\alpha$ " mode, the SOLEIL electron optics is tuned to decrease the momentum compaction factor $\alpha$ [24]. The lower the parameter $\alpha$, the narrower the spatial distribution of electrons, and the shorter the X-ray pulses. In addition, the current per electron bunch is reduced in order to avoid an increase of the electron bunch lengths due to electron-electron repulsion.

SOLEIL now operates in its low-alpha mode 4 times a year during $48 \mathrm{~h}$ sessions. For the present experiment, we worked with a hybrid filling pattern consisting of $312+1$ electron bunches carrying each $65 \mu \mathrm{A}$ and a value of $\alpha_{l}=1.710^{-5}$ (nominal $\alpha / 25$ ). The X-ray pulse duration measured in these conditions is $5 \mathrm{ps}$ rms $(\sim 12$ ps FWHM $)[4]$.

\subsection{Pump-probe diffraction setup at CRISTAL beamline}

The laser used for optical pumping is composed of a Ti:sapphire mode-locked oscillator coupled with a regenerative Ti:sapphire amplifier (Micra $5 \mathrm{~W}+$ Legend Elite Duo USX from Coherent). It produces 41 fs pulses (FWHM, measured value) of $800 \mathrm{~nm}$ wavelength with a tunable repetition rate ( 1 to $10 \mathrm{kHz})$. The energy per pulse is 5 $\mathrm{mJ}$ at $1 \mathrm{kHz}$, and $500 \mu \mathrm{J}$ at $10 \mathrm{kHz}$. The laser is installed in a dedicated hutch close to the beamline, in order to ensure a clean and temperature-controlled environment. The laser beam is transferred to the X-ray radiation shielded hutch through a tube line, and a chicane with two successive upward and sideways deviations. The total length of the beam path from the laser output to the sample is about $17 \mathrm{~m}$.

The $\mathrm{kHz}$ repetition rates on the pump laser side are well adapted for pump-probe experiments, because the heat deposited by the laser pulse dissipates in a few ms in a solid. On the other hand, synchrotron X-ray pulses are generally produced at $\mathrm{MHz}$ frequencies. In order to perform pump-probe experiments, one has to select only one X-ray pulse after each laser excitation, which is achieved with the XPAD3.2 detector [25]. In this detector, the incoming photons interact with a silicon sensor whose backside is pixelated. In each pixel, the charge detection stage handles the incoming photons continuously, but the counter can be enabled or disabled with a logic gate. Working frequencies up to $100 \mathrm{kHz}$ and gate durations as short as $90 \mathrm{~ns}$ are supported. This allows a flexible choice of the repetition rate and the X-ray pulse selection pattern (either single pulse or macro-pulse formed by several single pulses).

The pump laser and the XPAD3.2 detector are synchronized to the $352.202 \mathrm{MHz}$ radiofrequency signal $(\mathrm{RF})$ and phased to the $846.639 \mathrm{kHz}$ storage ring clock, via a FPGA-based custom made synchronization board [26]. During the set up of the pump-probe experiment, the logic gate of XPAD3.2 detector is first phased with the arrival time of a chosen X-ray-pulse, from a measurement of the intensity scattered by a piece of teflon as a function of the applied time delay. In a second step, the pump-probe delay has to be determined, which is defined as the difference in arrival times between the laser pulse and the X-ray pulse selected by the XPAD3.2 detector. For this purpose, a InGaAs metal-semiconductor-metal photodiode (G7096-03 from Hamamatsu) is placed at sample position and used to monitor both the X-ray and laser pulse arrival times [27] on a $3 \mathrm{GHz}$ digital oscilloscope (DSO80304B from Agilent). By triggering the oscilloscope with a copy of the logic gate, the X-ray pulse selected by the XPAD3.2 detector can be readily identified, and the actual pumpprobe delay measured. Let us note that SOLEIL storage ring is operated in such a way that the isolated bunch used for pump-probe experiments is always located in the same RF bucket. As a consequence, the detector and laser delay settings remain similar whatever the mode of operation used, depending only slightly on the detector position and the placement of a few laser optics upstream from the sample. 


\subsection{Experimental details}

The $200 \mathrm{~nm}$ thick, (111)-oriented thin film of Bi was synthesized in two steps. First, $\mathrm{Bi}(110)$ was deposited on a (100)-oriented $\mathrm{KCl}$ substrate following the method described in Ref. [28]. The $\mathrm{Bi}(110)$ was then annealed at the temperature of $250^{\circ} \mathrm{C}$ in a $\mathrm{N}_{2}$ atmosphere, which lead to a change in the film orientation from (110) to (111). The actual orientation was checked afterwards by X-ray diffraction.

We have studied the 111 Bragg reflection in a horizontal scattering plane geometry, with the laser beam in this plane and perpendicular to the X-ray beam. Diffraction was observed at the $\theta_{111} \simeq 9.005^{\circ}$ angle, thus implying a laser angle of incidence of $\sim 9.005^{\circ}$ from the normal to the sample surface.

The Bi thin-film was excited by $800 \mathrm{~nm}, 204 \mu \mathrm{J}$ laser pulses with a linear horizontal polarization at a $1 \mathrm{kHz}$ repetition rate. The laser beam size was determined by scanning across a knife-edge, in front of a powermeter. This measurement yielded a beam diameter of $2.4 \mathrm{~mm}$ FWHM. The sample was thus excited at a fluence of $4.5 \mathrm{~mJ} / \mathrm{cm}^{2}$, from which we estimate the absorbed fluence to $2.4 \mathrm{~mJ} / \mathrm{cm}^{2}$. Following Johnson et al. [22], we take the penetration depth of the $800 \mathrm{~nm}$ laser pulses into the Bi thin-film as equal to $\zeta=26 \mathrm{~nm}$.

The incident X-ray energy was set to $10.026 \mathrm{keV}\left(\Delta E / E \sim 10^{-4}\right)$. At this energy and nominal $\alpha / 25$ (see Sec. 2.1), the number of photons per pulse at sample position is estimated to 2300. Taking into account the angle of incidence, the penetration depth of X-rays into the Bi thin-film was $1.2 \mu \mathrm{m}$, i.e. the whole thickness of the film was probed. The X-ray beam size was set to $50 \times 120 \mu \mathrm{m}^{2}(\mathrm{H} \times \mathrm{V})$ with slits, resulting in a footprint of $300 \times 120 \mu \mathrm{m}^{2}$ onto the sample surface.

The ultrafast pump laser pulse generates a longitudinal acoustic pulse which bounces back and forth inside the $\mathrm{Bi}$ thin-film. The time-dependent, average longitudinal strain is $\eta_{3}=\Delta d_{111}(t) / d_{111}$, where $d_{111}$ is the interplanar (111) distance before laser excitation. In the present experiment, $d_{111}$ was measured as a function of pump-probe delay $t$, by performing $\theta$-scans through the (111) reciprocal position.

\section{Results}

Fig. 1a) shows a $\theta$-scan performed on the unpumped sample with the XPAD3.2 logic gate deactivated (i.e., all the photons were counted without timing constraints). The FWHM of the (111) Bragg peak is found equal to $0.036^{\circ}$ attesting to the good quality of the film. The same $\theta$-scan with the logic gate of the detector activated is represented in Fig. 1b). As expected, the photon count rate decreases dramatically, despite more favorable acquisition parameters (exposure time $10 \mathrm{~s}$ and X-ray attenuation 50 [29]). Due to the limited time available for our measurements, the angular range of the $\theta$-scans was restricted to $0.0375^{\circ}$. The counting time was $20 \mathrm{~s}$ per $0.0025^{\circ}$ step, the $\mathrm{X}$-ray attenuation being set to 10 . The $\theta$-scan measured in the latter conditions on the pumped Bi thin-film and at pump-probe delay 0 is represented in Fig. 1c).

All $\theta$-scans were fitted with a lorentzian-shaped function plus a $y_{0}$ background, estimated from complete $\theta$-scans (see fig. 1b). The integrated intensity of the 111 peak is found independent of the pump-probe delay within the $10 \%$ error bars. On the other hand, both the angular position $\theta_{0}$ and the FWHM of the peaks exhibit significant variations as a function of time after laser excitation.

The dynamics found for $\theta_{0}$ is reported in fig. 2. We note $\Delta \theta(t)$ the difference between the observed Bragg angle and its average value at negative delays $\theta_{0}^{-}$. The average longitudinal strain given on the right scale was calculated from the relations $\eta_{3}=\Delta d_{111}(t) / d_{111}=-\Delta \theta(t) / \tan \theta_{0}^{-}$. As the pump-probe delay becomes positive, the Bragg angle first rapidly decreases $(0 \leq t \leq 80 \mathrm{ps})$, and then oscillates around a 


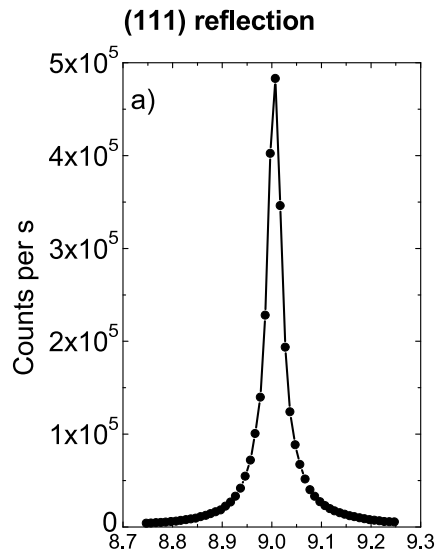

$\theta[9$

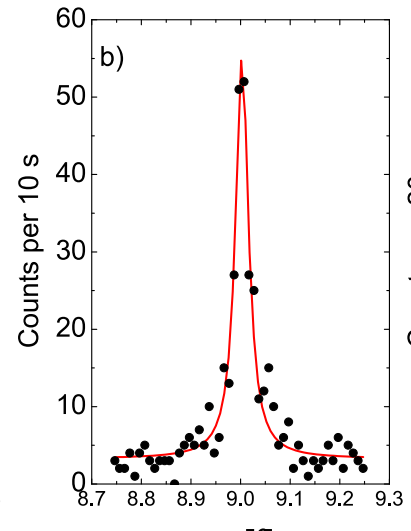

$\theta[9$

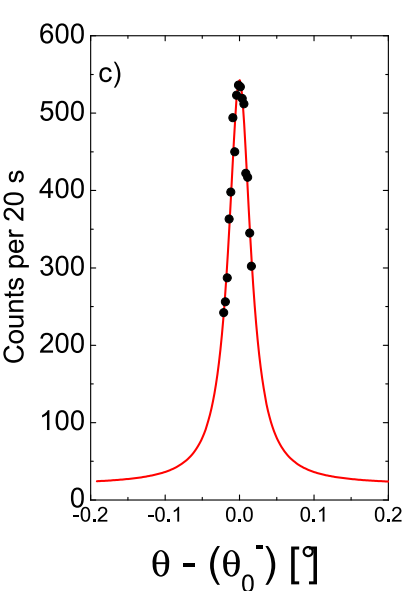

Fig. 1. 111 Bragg reflection intensity as a function of $\theta$. a) Static measurement without $\mathrm{X}$-ray pulse selection [counting time $1 \mathrm{~s}$, X-ray attenuation factor 100]. b) $\theta$-scan taken in the gated mode of the detector, with the pump laser OFF [counting time $10 \mathrm{~s}$ and X-ray attenuation factor 50]. A lorentzian fit of the peak (red line) yields a reference for background noise. c) $\theta$-scan taken in the gated mode of the detector, with the pump laser ON at pumpprobe delay $t=0$ [counting time $20 \mathrm{~s}$, X-ray attenuation factor 10]. The peak is represented together with its lorentzian fit (red line).

value $0.005^{\circ}$ lower than before laser excitation. This behavior is consistent with the hypothesis that the laser pulse energy is converted into both heat, with subsequent increase of the unit cell parameters, and elastic energy, in the form of a propagating acoustic pulse. In order to extract the relevant physical parameters, we fitted the data by the following function:

$$
\left[A \cos \left(\left(t-t_{0}\right) \times 2 \pi / T\right) \times e^{-\left(t-t_{0}\right) / \tau}-A\right] \times\left[\operatorname{sgn}\left(t-t_{0}\right)+1\right] / 2,
$$

where $A$ is the amplitude of the angular shift, and $t_{0}$ the time corresponding to the onset of its dynamics. $T$ and $\tau$ represent the period and the damping constant of the acoustic pulse oscillation in the film. We obtained the following fitted parameters: $A=0.0049^{\circ}, t_{0}=-17.6 \mathrm{ps}, T=207.5 \mathrm{ps}$, and $\tau=208.7 \mathrm{ps}$.

The 111 reflection FWHM, which equals $0.036^{\circ}$ at negative delays, jumps to $0.047^{\circ}$ at delays in the range $[0-80 \mathrm{ps}]$ and then drops back to $\sim 0.039^{\circ}$ for all larger delays. Large error bars of $8 \%$ prevent us from giving a more precise description of the FWHM dynamics, yet we can conclude that a mosaic disorder sets in during the first $80 \mathrm{ps}$ after laser excitation.

\section{Discussion}

In order to discuss these results and observations, let us recall the basic theory of propagation of strain pulses, first introduced by Thomsen et al. [23]. In its simplest form, this theory states that after electron relaxation, which is about $10 \mathrm{ps}$ in bismuth [20], the energy deposited by the laser pulse gives a $z$-dependent temperature rise $\Delta T(z)$, governed by the laser penetration length $\zeta$. This temperature increase gives rise to a negative, $z$-dependent normal stress $\sigma_{3}$. By taking explicitely into account 


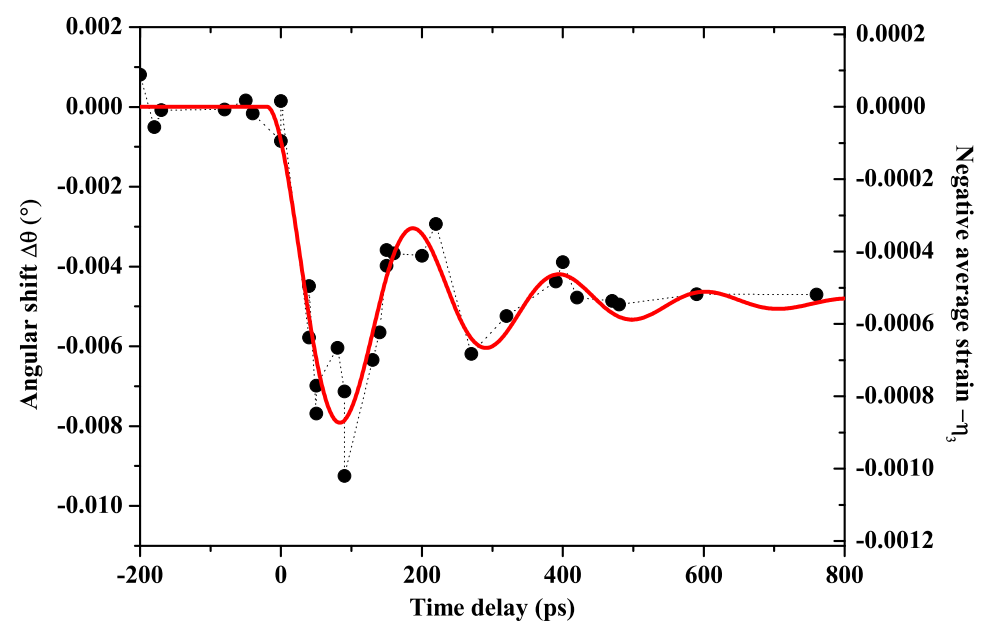

Fig. 2. Variation of the rocking curve maximum angle of the 111 Bragg reflection as a function of the time delay. The red curve is a fit as described in the text.

the bismuth anisotropy, this stress can be expressed as:

$$
-\left(c_{13} \alpha_{1}+c_{23} \alpha_{2}+c_{33} \alpha_{3}\right) \Delta T(z)
$$

where $c_{i j}$ are the elastic constants and $\alpha_{i}$ the linear thermal expansion coefficients. Note that in this formula, the 1,2 and 3 indices correspond to the $[1 \overline{1} 0],[11 \overline{2}]$ and [111] rhomboedral directions, respectively.

The total stress is thus expressed as

$$
\sigma_{3}(z)=c_{33} \eta_{3}(z)-\left(c_{13} \alpha_{1}+c_{23} \alpha_{2}+c_{33} \alpha_{3}\right) \Delta T(z),
$$

where $\eta_{3}(z)$ is the normal strain. This stress will relax thanks to a strain (or acoustic) pulse of width $2 \zeta$, propagating in the film with the longitudinal sound velocity given by

$$
\rho v_{L}^{2}=c_{33},
$$

where $\rho=9.8 \mathrm{~g} / \mathrm{cm}^{3}$ is the bismuth mass density [30]. In a thin film, this pulse is reflected by both surfaces, giving rise to an oscillation of the average normal strain, the period of which being equal to $2 L / v_{L}$. Using $L=200 \mathrm{~nm}$ and the value of $v_{L}=1972 \mathrm{~m} / \mathrm{s}$ given in ref. [30], we predict an oscillation period of $202.8 \mathrm{ps}$. The experimental value of $T=207.5 \mathrm{ps}$ is consistent with the latter value, within the time resolution of $10 \mathrm{ps}$.

Following ref. [23], if we neglect the heat diffusion, the strain pulse dimension remains equal to $2 \zeta$, which is much smaller than the film width. Thus, when the pulse is in the middle of the film, the lattice contraction compensate for the lattice expansion and the average strain should not vary. This is clearly not consistent with the regular cosine variation we observe. However, according to [22], the thermal diffusivity $D$ constant is equal to $2.3 \mathrm{~cm}^{2} / \mathrm{s}$, which gives a typical time $L^{2} / D \simeq 175 \mathrm{ps}$ for the heat to diffuse through the film. This time has the same order of magnitude than the oscillation period, which means that the heat diffusion cannot be neglected whilst the pulse propagates. One can equivalently calculate $\frac{D}{v_{L} \zeta} \simeq 4.5$, a large value indicating that heat conduction has a large effect on the stress relaxation [23]. These arguments 
give a qualitative explanation of the smooth cosine variation of the average normal strain we observe.

Our measurements also give the opportunity to calculate the effective temperature increase of the film. According to our data, the stress is completely relaxed after $\sim 800 \mathrm{ps}$. This allows one to calculate the average temperature increase $\overline{\Delta T}$ from the measured average normal strain $\overline{\eta_{3}}$. Indeed, setting $\sigma_{3}(z)=0$ leads to:

$$
\overline{\Delta T}=\frac{c_{33}}{c_{13} \alpha_{1}+c_{23} \alpha_{2}+c_{33} \alpha_{3}} \overline{\eta_{3}} \text {. }
$$

From the measured sound velocity $\left(v_{L}=1927.7 \mathrm{~m} / \mathrm{s}\right)$, we find $c_{33}=36.4 \mathrm{GPa}$. Assuming $\alpha_{1} \sim \alpha_{2}$ and using $\alpha_{1}=11.8 \times 10^{-6}, \alpha_{3}=17.7 \times 10^{-6}$ [31], and $c_{12}=24.7 \mathrm{GPa}$, $c_{13}=24.5 \mathrm{GPa}[30]$, one finds $\overline{\Delta T} \simeq 30,000 \overline{\eta_{3}}$. With a saturated value of $\overline{\eta_{3}} \sim 0.005$, the increase in temperature is $15 \mathrm{~K}$.

This value is to be compared to the increase in temperature expected from the fluence of the laser. This increase is equal to the density of energy deposited divided by the volumic heat capacity $\left(C=1.2 \times 10^{6} \mathrm{~J} \cdot \mathrm{m}^{-3} \cdot \mathrm{K}^{-1}\right)$. By using the value of $\S 2.3$, one finds an expected increase of about $100 \mathrm{~K}$. The difference between the two values indicates that a large part of the deposited energy spreads outside the laser irradiated zone of the film, either in the substrate (transmitted strain pulses), or in the sides of the films (heat diffusion).

In conclusion, we have observed the laser-induced normal strain oscillations in a $200 \mathrm{~nm}$ bismuth thin film, which qualitatively agrees with current theories. Nevertheless, a complete comprehension of the phenomena would require additional theoretical work, taking into account the heat diffusion, strain pulse damping and reflection at interfaces.

This work was supported by Rennes Metropole and the ANR (09-BLAN-0212 project). We are indebted to Marie-Agnès Tordeux, Philippe Hollander, Stéphanie Hustache and JeanPaul Ricaud for their contribution in this work.

\section{References}

1. M. Lorenc, J. Hébert, N. Moisan, E. Trzop, M. Servol, M. Buron-Le Cointe, H. Cailleau, M.L. Boillot, E. Pontecorvo, M. Wulff, S. Koshihara, and E. Collet, Phys. Rev. Lett. 103, (2009) 028301.

2. J. Feikes, K. Holldack, P. Kuske, and G. Wüstefeld, Proceedings of EPAC 2004, (2004) 1951.

3. P. Brunelle, F. Briquez, A. Loulergue, O. Marcouillé, A. Nadji, L.S. Nadolski, M.-A. Tordeux, and J. Zhang, Proceedings of IPAC 2011, (2011) 2124.

4. M.-A. Tordeux, J. Barros, A. Bence, P. Brunelle, N. Hubert, M. Labat, A. Nadji, L. Nadolski, P. Lebasque, J.-P. Pollina, C. Evain, Proceedings of IPAC 2012, (2012) 1608.

5. A. Zholents, P. Heimann, M. Zolotorev, and J. Byrd, Nuclear Instruments and Methods A 425, (1999) 385.

6. R.W. Schoenlein, S. Chattopadhyay, H.H.W. Chong, T.E. Glover, P.A. Heimann, C.V. Shank, A.A. Zholents, M.S. Zolotorev, Science 287, (2000) 2237.

7. P. Beaud, S. L. Johnson, A. Streun, R. Abela, D. Abramsohn, D. Grolimund, F. Krasniqi, T. Schmidt, V. Schlott, and G. Ingold, Phys. Rev. Lett. 99, (2007) 174801.

8. C. Rischel, A. Rousse, I. Uschmann, P.-A. Albouy, J.-P. Geindre, P. Audebert, J.-C. Gauthier, E. Forster, J.-L. Martin, A. Antonetti, Nature 390, (1997) 490.

9. S. Kneip, C.McGuffey, J. L.Martins, S. F.Martins, C. Bellei, V. Chvykov, F. Dollar, R. Fonseca, C. Huntington, G. Kalintchenko, A.Maksimchuk, S. P. D.Mangles, T.Matsuoka, S. R. Nagel, C. A. J. Palmer, J. Schreiber, K. Ta Phuoc, A. G. R. Thomas, V. Yanovsky, L. O. Silva, K. Krushelnick, and Z. Najmudin, Nature Physics 6, (2010) 980. 
10. A. Cho, Science 330, (2010) 1470.

11. P. Emma, R. Akre, J. Arthur, R. Bionta, C. Bostedt, J. Bozek, A. Brachmann, P. Bucksbaum, R. Coffee, F. J. Decker, Y. Ding, D. Dowell, S. Edstrom, A. Fisher, J. Frisch, S. Gilevich, J. Hastings, G. Hays, Ph. Hering, Z. Huang, R. Iverson, H. Loos, M. Messerschmidt, A. Miahnahri, S. Moeller, H. D. Nuhn, G. Pile, D. Ratner, J. Rzepiela, D. Schultz, T. Smith, P. Stefan, H. Tompkins, J. Turner, J. Welch, W. White, J. Wu, G. Yocky, and J. Galayda, Nature Photonics 4, (2010) 641.

12. J. Larsson, A; Allen, P.H. Bucksbaum, R.W. Falcone, A. Lindenberg, G. Naylor, T. Missalla, D.A. Reis, K. Scheidt, A. Sjrgen, P. Sondhauss, M. Wulff, and J.S. Wark, Appl. Phys. A 75, (2002) 467-478.

13. Christoph Rose-Petruck, Ralph Jimenez, Ting Guo, Andrea Cavalleri, Craig W. Siders, Ferenc Ráksi, Jeff A. Squier, Barry C. Walker, Kent R. Wilson, and Christopher P. J. Barty, Nature 398, (1999) 310.

14. Jie Chen, Wei-Kan Chen, Jau Tang, and Peter M. Rentzepis, Proc. Natl. Acad. Sci. U.S.A. 108, (2011) 18887.

15. Matthieu Nicoul, Uladzimir Shymanovich, Alexander Tarasevitch, Dietrich von der Linde, and Klaus Sokolowski-Tinten, Appl. Phys. Lett. 98, (2011) 191902.

16. Bismuth has a rhomboedral A7 structure (space group R $\overline{3} m$ ) with the following rhomboedral (hexagonal) parameters at $300 \mathrm{~K}: a_{R}=4.746 \AA, \alpha_{R}=57.23^{\circ}\left(a_{H}=4.546 \AA\right.$, $\left.c_{H}=11.862 \AA\right)$. In this article, we refer to the rhomboedral cell which contains $2 \mathrm{Bi}$ atoms located on the (2c) Wyckoff positions.

17. T.K. Cheng, S.D. Brorson, A.S. Kazeroonian, J.S. Moodera, G. Dresselhaus, M.S. Dresselhaus, and E.P. Ippen, Appl. Phys. Lett. 57, (1990) 1004.

18. K. Sokolowski-Tinten, C. Blome, J. Blums, A. Cavalleri, C. Dietrich, A. Tarasevitch, I. Uschmann, E. Frster, M. Kammler, M. Horn-von-Hoegen, and D. von der Linde, Nature 422, (2003) 287.

19. D.M., Fritz, D.A. Reis, B. Adams, R.A. Akre, J. Arthur, C. Blome, P.H. Bucksbaum, A.L. Cavalieri, S. Engemann, S. Fahy, R.W. Falcone, P.H. Fuoss, K.J. Gaffney, M.J. George, J. Hajdu, M.P. Hertlein, P.B. Hillyard, M. Horn-von Hoegen, M. Kammler, J. Kaspar, R. Kienberger, P. Krejcik, S.H. Lee, A.M. Lindenberg, B. McFarland, D. Meyer, T. Montagne, E.D. Murray, A.J. Nelson, M. Nicoul, R. Pahl, J. Rudati, H. Schlarb, D.P. Siddons, K. Sokolowski-Tinten, Th. Tschentscher, D. von der Linde, and J.B. Hastings, Science 315, (2007) 633.

20. S. L. Johnson, P. Beaud, E. Vorobeva, C. J. Milne, E. D. Murray, S. Fahy, and G. Ingold, Acta Cryst. A 66, (2010), 157.

21. H.J. Zeiger, J. Vidal, T.K. Cheng, E.P. Ippen, G. Dresselhaus, and M.S. Dresselhaus, Phys. Rev. B 45, (1992) 768.

22. S. L. Johnson, P. Beaud, C. J. Milne, F. S. Krasniqi, E. S. Zijlstra, M. E. Garcia, M. Kaiser, D. Grolimund, R. Abela, and G. Ingold, Phys. Rev. Lett. 100, (2008) 155501.

23. C. Thomsen, H. T. Grahn, H. J. Maris, and J. Tauc, Phys. Rev. B 34, (1986) 4129.

24. The momentum compaction factor $\alpha$ relates the length of an electron trajectory $\left(L_{0}+\right.$ $\Delta L)$ to its difference in momentum $(\Delta P)$ with an synchronous electron $\left(L_{0}, P_{0}\right)$ :

$$
\alpha=\frac{\Delta L / L_{0}}{\Delta P / P_{0}}
$$

25. P. Pangaud, S. Basolo, N. Boudet, J.-F. Berar, B. Chantepie, J.-C. Clemens, P. Delpierre, B. Dinkespiler, K. Medjoubi, S. Hustache, M. Menouni, Ch. Morel, Nucl. Instrum. Methods Phys. Res. A 591, (2008) 159.

26. J.-P. Ricaud, P. Betinelli-Deck, J. Bisou, X. Elattaoui, C. Laulhé, P. Monteiro, L.S. Nadolski, G. Renaud, S. Ravy, M. Silly, F. Sirotti, Proceedings of ICALEPCS 2011, (2011) 1036 .

27. The spectral response range specified for this photodiode is 850 to $1650 \mathrm{~nm}$, but the detection efficiency is still sufficient to detect synchrotron X-ray pulses even in the low- $\alpha$ mode. The guard glass in front of the diode can be removed to increase sensitivity to $\mathrm{X}$-rays below $10 \mathrm{keV}$. 
28. G. Moriena, M. Hada, G. Sciaini, J. Matsuo, and R.J.D. Miller, J. Appl. Phys. 111, (2012) 043504.

29. For diffraction experiments, the XPAD3.2 detector has to be used in its proportional counting regime, i.e. with a number of incoming photons limited to 1 photon per pixel per 90 ns period. In some cases, especially when using hybrid filling modes, it is not possible to remove all X-ray attenuators despite a low counting rate in the time-resolved mode.

30. Y. Eckstein, A. W. Lawson, and D. H. Reneker, J. Appl. Phys. 31, (1960) 1534.

31. E. F. Cave and L. V. Holroyd, J. Appl. Phys. 31, (1960) 1357. 